\title{
On the Role of Membrane Structural Defects in Smith-Lemli-Opitz Syndrome \\ Fliesler SJ*
}

Research Service, VA Western New York Healthcare System, The Departments of Ophthalmology and Biochemistry, University at Buffalo- The State University of New York, and the SUNY Eye Institute

Smith-Lemli-Opitz syndrome (SLOS) is an autosomal recessive disease initially caused by mutations in the DHCR7 gene (OMIM\# 602858); this gene encodes the penultimate enzyme in the cholesterol biosynthetic pathway, 7-dehydrocholesterol reductase (3ß-hydroxysterol- $\Delta 7$-reductase; EC 1.3.1.21) [1,2]. Such mutations give rise to a catalytically defective enzyme, resulting in an inefficient conversion of 7-dehydrocholesterol (7DHC), the immediate biogenic precursor of cholesterol, to cholesterol. This causes aberrant accumulation of 7DHC (and, typically to a far lesser extent, its isomer, 8-dehydrocholesterol (8DHC)) and reduced levels of cholesterol in bodily tissues and fluids [3]. [Notably, there are no reports of an "all-or-none" effect, where cells or tissues from affected individuals or unborn fetuses contain no detectable residual cholesterol. More typically, the cholesterol levels are far below normal, while the dehydrosterol precursors are the dominant sterol species present.] The biological consequences of this biochemical defect, unlike many other monogenic diseases, can vary dramatically, with the severity of phenotypic abnormalities ranging from relatively mild to severe, even including embryonic or early neonatal lethality [1]. SLOS is considered a pediatric disorder, since the disease manifests in early childhood and few affected individuals survive beyond the teenage years.

There has been considerable speculation over the years about exactly why this congenital enzymatic defect could lead to such a profound disease. One obvious culprit that has been widely considered is a lack of sufficient cholesterol during early embryogenesis, particularly during the formation of the nervous system $[1,4]$. However, this presupposes that the level of total sterols is significantly less, particularly in nervous tissue, than normal and that the biological function(s) of cholesterol cannot be replaced adequately by the aberrant dehydrosterols that accumulate in this disease. With regard to the first aspect, while blood total sterol levels typically are far less than normal in SLOS patients, as well as in animal models of the disease, this is not usually the case for the brain or other tissues, e.g., when total sterols are normalized to tissue wet weight [1-3]. However, there is little comparable information available regarding human or animal embryos.

With regard to the second aspect, this begs the question: why not? For the purposes of this brief editorial, and considering the topical scope of this particular journal, I will restrict my remarks to the chemistry of sterols and the role sterols play as structural components of biological membranes. However, the reader should appreciate that cholesterol serves many biological functions in addition to its role as a membrane constituent, including as an obligatory precursor for steroid hormones and bile acids, and as an essential covalent adduct requisite for the biological activity of the hedgehog family of morphogens [5-7].

Cholesterol and its immediate biogenic precursor, 7DHC, are both 27 -carbon, $3 \beta$-monohydroxy sterols, differing from one another by just one double bond: 7DHC contains two double bonds, i.e., $\Delta 5$ (between C5-C6 in ring B) and $\Delta 7$ (between $\mathrm{C} 7-\mathrm{C} 8$ in ring $\mathrm{B}$ ) in the sterol nucleus, whereas cholesterol has only one, the $\Delta 5$ double bond. On first principles, despite a slight "pucker" in the otherwise planar fused sterol ring structure, the extra double bond in 7DHC would not be expected to represent a significant physical perturbation compared to the structure of cholesterol. Indeed, both have comparable melting temperatures (cholesterol, $148.5^{\circ} \mathrm{C}$; $7 \mathrm{DHC}, 151-152^{\circ} \mathrm{C}$ ) and densities (cholesterol, $1.07 \mathrm{~g} / \mathrm{cm}^{3}$; 7DHC, $1.00 \mathrm{~g} / \mathrm{cm}^{3}$ ) [8]. Furthermore, studies employing model membranes, e.g., Langmuir monolayer films composed of sterolglycerophospholipid mixtures spread on an aqueous interface, also have shown that 7DHC and cholesterol exhibit very similar physical properties, including film compressibility and molecular areas [9-11].

Sterols in biological membranes are not distributed uniformly; rather, they tend to aggregate in "lipid rafts": transient, highly-ordered microdomains enriched in sterols and sphingolipids, compared to the bulk phase, which are known to serve as platforms for signal transduction [12,13]. So, the question arises: maybe 7DHC is not able to form lipid rafts as well as does cholesterol? However, as independent studies have clearly demonstrated, this is not the case; in fact, if anything, 7DHC promotes lipid raft formation even slightly better than does cholesterol [14-16]. A subsequent study by Kavarova et al. [17], using mast cells derived from Dhcr7-knockout mice, suggested that 7DHC might actually disrupt lipid raft organization and function. It should be noted, however, that the latter is based upon interpretation of the primary data; the authors did not directly measure lipid raft lifetimes, nor do reciprocal experiments systematically removing and then replacing the endogenous membrane sterols with exogenous, highly purified 7DHC, in addition to the comparable experiments they did perform using methyl- $\beta$-cyclodextrin and cholesterol. Also, 7DHC represented, at most, about $30 \mathrm{~mol} \%$ of total sterols in the Dhcr7knockout cells, and viability in culture over a 120-hour duration was only modestly (ca. 7\%) decreased, compared to wild type controls. Hence, it's not clear that the presence of 7DHC, per se, in the lipid raft domains caused the observed effects. Tulenko and colleagues [18], using skin fibroblasts from SLOS patients, showed that those cells contained elevated 7DHC and reduced cholesterol levels (although total sterols were only modestly reduced, and 7DHC was only about $20 \%$ of total), as well as altered (reduced) membrane fluidity, and dramatically altered ion permeability, enzymatic, and signal transduction capacities, relative to normal control cells. They interpreted their results to signify that "disturbance in membrane sterol content in SLOS, likely at the level of membrane caveolae, directly contributes to the widespread tissue abnormalities in this disease" [18]. However, additional changes in

*Corresponding author: Fliesler SJ, VA Western New York Healthcare System, USA, Tel: 716 862-6538; Fax: 716 862-6526; E-mail: fliesler@buffalo.edu

Received December 07, 2013; Accepted December 07, 2013; Published December 09, 2013

Citation: Fliesler SJ (2013) On the Role of Membrane Structural Defects in Smith-Lemli-Opitz Syndrome. J Membra Sci Technol 3: e119. doi:10.4172/21559589.1000e119

Copyright: (c 2013 Fliesler SJ. This is an open-access article distributed under the terms of the Creative Commons Attribution License, which permits unrestricted use, distribution, and reproduction in any medium, provided the original author and source are credited. 
Citation: Fliesler SJ (2013) On the Role of Membrane Structural Defects in Smith-Lemli-Opitz Syndrome. J Membra Sci Technol 3: e119. doi:10.4172/2155-9589.1000e119

Page 2 of 3

lipid composition other than sterols, which may have had a significant impact on the measurements, were not assessed in either of those two studies. This is important, since studies using the AY9944 rat model of SLOS have demonstrated marked alterations in fatty acid composition of whole retina and isolated rod outer segment membranes, particularly a dramatic reduction in the mol\% of their major fatty acyl constituent, docosahexaenoic acid (DHA), with concomitant changes in membrane fluidity $[19,20]$. A more recent study from Ren and colleagues [21], again using skin fibroblasts from SLOS patients as well as model membranes, has provided evidence suggesting that altered membrane sterol composition can provoke associated protein changes in caveolae that, in turn, can significantly impact caveolae-dependent signaling (although the authors did point out that caveolar ultrastructure, per se, was not altered, relative to controls). Again, no assessment of other (non-sterol) lipid compositional changes was performed, and the authors conceded that "additional cellular alterations beyond mere changes associated with abnormal sterols in the membrane likely contribute to the pathogenesis of SLOS" [21].

Studies on the chemistry of 7DHC have shown that it is potentially the most highly oxidizable organic molecule known to date [22], remarkably nearly seven times more so than DHA (which has six double bonds, compared to 7DHC's two). In fact, oxidation of 7DHC can give rise to more than a dozen, chemically distinct oxysterol derivatives, some of which are horrendously toxic to cells $[23,24]$. Such compounds have been detected readily in cells, tissues, and biological fluids from SLOS patients and from animal models of SLOS [25-29]. It is well known that oxysterols, in general, do not integrate into membrane bilayers in a manner comparable to that of cholesterol; in fact, they tend to disrupt the packing order of the glycerophospholipids that constitute the bulk phase of the bilayer [30,31]. Given these findings, it is possible that at least some of the biological and biophysical effects observed in prior investigations relevant to SLOS were due to in situ formation of 7DHCderived oxysterols. In fact, cytotoxic, 7DHC-derived oxysterols may be key players underlying the pathobiology of SLOS [32,33]. Hence, in addition to cholesterol supplementation or interventions that target the aberrant formation and accumulation of 7DHC, which to date have not been shown to be reliably or substantially efficacious in minimizing SLOS-associated phenotypic or functional abnormalities (for a review, see $[1,34])$, an improved therapeutic approach might include antioxidants (in addition to cholesterol) to suppress the formation of 7DHC-derived oxysterols $[32,33,35]$. Such an approach is currently ongoing in a limited clinical trial at Children's Hospital Denver, and the initial results are showing promise (R. Braverman and E. Elias, personal communication).

\section{Acknowledgment}

Supported in part, by $\mathrm{NIH/NEI}$ grant RO1EY007361, by an Unrestricted Grant to the Department of Ophthalmology, University at Buffalo, from Research to Prevent Blindness, and by facilities and resources provided by the Veterans Administration Western New York Healthcare System. The opinions expressed herein do not necessarily reflect those of the Veterans Administration or the U.S. Government.

\section{References}

1. Porter FD (2008) Smith-Lemli-Opitz syndrome: pathogenesis, diagnosis and management. Eur J Hum Genet 6: 535-541.

2. Correa-CLS, Porter FD (2005) 3 beta-hydroxysterol delta 7-reductase and the Smith-Lemli-Opitz syndrome. Mol Genet Metab 84: 112-126.

3. Tint GS, Irons M, Elias ER, Batta AK, Frieden R, et al. (1994) Defective cholesterol biosynthesis associated with the Smith-Lemli-Opitz syndrome. N Engl J Med 330: 107-113.

4. Porter FD, Herman GE (2011) Malformation syndromes caused by disorders of cholesterol synthesis. J Lipid Res 52: 6-34.
5. Yeagle PL (1988) Biology of Cholesterol. Taylor \& Francis, CRC press, Boca Raton.

6. Myant NB (1981) The Biology of Cholesterol and Related Steroids. Heinemann Press, London.

7. Beachy PA, Cooper MK, Young KE, von Kessler DP, et al. (1997) Multiple roles of cholesterol in hedgehog protein biogenesis and signaling. Cold Spring Harb Symp Quant Biol 62: 191-204.

8. Haynes WM (Ed) (2013) CRC Handbook of Chemistry and Physics (94th edn) Taylor \& Francis, CRC Press, Boca Raton.

9. Serfis AB, Brancato S, Fliesler SJ (2001) Comparative behavior of sterols in phosphatidylcholine-sterol monolayer films. Biochim Biophys ActaBiomembranes 1511: 341-348.

10. Berring EE, Borrenpohl K, Fliesler SJ, Serfis AB (2005) A comparison of the behavior of cholesterol and selected derivatives in mixed sterol-phospholipid Langmuir monolayers: A fluorescence microscopy study. Chem Phys Lipids 136: 1-12.

11. Lintker BK, Kpere-Daibo P, Fliesler SJ, Serfis AB (2009) A comparison of the packing behavior of egg phosphatidylcholine with cholesterol and biogenically related sterols in Langmuir monolayer films. Chem Phys Lipids 161: 22-31.

12. Brown DA, London $E$ (1998) Functions of lipid rafts in biological membranes. Annu Rev Cell Dev Biol 14: 111-136.

13. Sonnino S, Prinetti A (2013) Membrane domains and the "lipid raft" concept Curr Med Chem 20: 4-12.

14. Xu X, London E (2000) The effect of sterol structure on membrane lipid domains reveals how cholesterol can induce lipid domain formation. Biochemistry 39: 843-849.

15. Xu X, Bittman R, Duportail G, Heissler D, et al. (2001) Effect of the structure of natural sterols and sphingolipids on the formation of ordered sphingolipid/ sterol domains (rafts): Comparison of cholesterol to plant, fungal, and diseaseassociated sterols and comparison of sphingomyelin, cerebrosides, and ceramide. J Biol Chem 276: 33540-33546.

16. Keller RK, Arnold TP, Fliesler SJ (2004) Formation of 7-dehydrocholesterolcontaining membrane rafts in vitro and in vivo, with relevance to the SmithLemli-Opitz syndrome. J Lipid Res 45: 347-355.

17. Kovarova M, Wassif CA, Odom S, Liao K, et al. (2006) Cholesterol deficiency in a mouse model of Smith-Lemli-Oitz syndrome revelas increased masst cell responsiveness. J Exp Med 203: 1161-1171.

18. Tulenko TN, Boeze-Battaglia K, Mason RP, Tint GS, et al. (2006) A membrane defect in the pathogenesis of the Smith-Lemli-Opitz syndrome. J Lipid Res 47 : 134-143.

19. Ford DA, Monda JK, Brush RS, Anderson RE, et al. (2008) Lipidomic analysis of the retina in a rat model of Smith-Lemli-Opitz syndrome: alterations in docosahexaenoic acid content of phospholipid molecular species. J Neurochem 105: 1032-1047.

20. Boesze-Battaglia K, Damek-Poprawa M, Mitchell DC, Greeley L, Brush RS et al. (2008) Alteration of retinal rod outer segment membrane fluidity in a rat model of Smith-Lemli-Opitz syndrome. J Lipid Res. 49: 1488-1499.

21. Ren G, Jacob RF, Kaulin Y, Dimuzio P, et al. (2011) Alterations in membrane caveolae and BKCa channel activity in skin fibroblasts in Smith-Lemli-Opitz syndrome. Mol Genet Metab 104: 346-355.

22. Xu L, Davis TA, Porter NA (2009) Rate constants for peroxidation of polyunsaturated fatty acids and sterols in solution and in liposomes. $\mathrm{J} \mathrm{Am}$ Chem Soc. 131: 13037-13044.

23. Xu L, Korade Z, Porter NA (2010) Oxysterols from free radical chain oxidation of 7-dehydrocholesterol: product and mechanistic studies. J Am Chem Soc 132: 2222-2232.

24. Korade Z, Xu L, Shelton R, Porter NA (2010) Biological activities of 7-dehydrocholesterol-derived oxysterols: implications for Smith-Lemli-Opitz syndrome. J Lipid Res 51: 3259-3269.

25. Xu L, Korade Z, Rosado DA Jr, Liu W, et al. (2011) An oxysterol biomarker for 7-dehydrocholesterol oxidation in cell/mouse models for Smith-Lemli-Opitz syndrome. J Lipid Res 52: 1222-1233.

26. Xu L, Liu W, Sheflin LG, Fliesler SJ, Porter NA (2011) Novel oxysterols observed in tissues and fluids of AY9944-treated rats: a model for Smith-LemliOpitz syndrome. J Lipid Res 52: 1810-1820. 
Citation: Fliesler SJ (2013) On the Role of Membrane Structural Defects in Smith-Lemli-Opitz Syndrome. J Membra Sci Technol 3: e119. doi:10.4172/2155-9589.1000e119

Page 3 of 3

27. Xu L, Sheflin LG, Porter NA, Fliesler SJ (2012) 7-Dehydrocholesterol-derived oxysterols and retinal degeneration in a rat model of Smith-Lemli-Opitz syndrome. Biochim Biophys Acta 1821: 877-883.

28. Korade Z, Xu L, Mirnics K, Porter NA (2013) Lipid biomarkers of oxidative stress in a genetic mouse model of Smith-Lemli-Opitz syndrome. $\mathrm{J}$ Inherit Metab Dis 36: 113-122

29. Liu W, Xu L, Lamberson CR, Merkens LS, et al. (2013) Assays of plasma dehydrocholesteryl esters and oxysterols from Smith-Lemli-Opitz syndrome patients. J Lipid Res 54: 244-253.

30. Massey JB (2006) Membrane and protein interactions of oxysterols. Curr Opin Lipidol 17: 296-301.
31. Olkkonen VM, Hynynen R (2009) Interactions of oxysterols with membranes and proteins. Mol Aspects Med 30: 123-133

32. Fliesler SJ (2010) Retinal degeneration in a rat model of Smith-Lemli-Opitz Syndrome: thinking beyond cholesterol deficiency. Adv Exp Med Biol 664: 481 489.

33. Korade Z, Xu L, Harrison FE, Ahsen R, et al. (2013) Antioxidant Supplementation Ameliorates Molecular Deficits in Smith-Lemli-Opitz Syndrome. Biol Psychiatry

34. DeBarber AE, Eroglu Y, Merkens LS, Pappu AS, Steiner RD (2011) SmithLemli-Opitz syndrome. Expert Rev Mol Med 13: e24.

35. Fliesler SJ (2013) Antioxidants: The missing key to improved therapeutic intervention in Smith-Lemli-Opitz syndrome? Hereditary Genet 2: 119. 